\title{
Fabrication and Characterization of PZN-4.5PT Inorganic Perovskites Nanoparticles Thin Films Deposited on P-Type Silicon Substrate
}

\author{
Rémi Ndioukane ${ }^{1 *}$, Moussa Touré1, Diouma Kobor ${ }^{1}$, \\ Laurence Motte ${ }^{2}$, Marcel Pasquinelli' ${ }^{3}$, Jeanne Solard ${ }^{4}$ \\ ${ }^{1}$ Laboratoire de Chimie et de Physique des Matériaux (LCPM), Université Assane Seck de Ziguinchor, Ziguinchor, Sénégal \\ ${ }^{2}$ Laboratory for Vascular Translational Science (LVTS), Université Paris 13, Bobigny, France \\ ${ }^{3}$ Institutut Matériaux Microélectronique Nanosciences de Provence (IM2NP), Aix-Marseille Université, Marseille, France \\ ${ }^{4}$ Centrale de Proximité en Nanotechnologies de Paris Nord, Institut Universitaire de Technologie de Villetaneuse, Paris, France \\ Email: *r.ndioukane1532@zig.univ.sn
}

How to cite this paper: Ndioukane, R., Touré, M., Kobor, D., Motte, L., Pasquinelli, M. and Solard, J. (2018) Fabrication and Characterization of PZN-4.5PT Inorganic Perovskites Nanoparticles Thin Films Deposited on P-Type Silicon Substrate. Journal of Modern Physics, 9, 259-272.

https://doi.org/10.4236/jmp.2018.92018

Received: October 10, 2017

Accepted: January 21, 2018

Published: January 24, 2018

Copyright $\odot 2018$ by authors and Scientific Research Publishing Inc. This work is licensed under the Creative Commons Attribution International License (CC BY 4.0).

http://creativecommons.org/licenses/by/4.0/

\begin{abstract}
This work involves an investigation of nanostructures, microelectronic properties and domain engineering of nanoparticles thin layers of $\mathrm{Pb}(\mathrm{Zn} 1 /$ $\left.3 \mathrm{Nb}_{2 / 3}\right) \mathrm{O}_{3}-\mathrm{PbTiO}_{3}(\mathrm{PZN}-\mathrm{PT})$ ferroelectric single crystals deposited on nanostructured silicon substrate. In this study, devices made from PZN-4.5PT nanoparticles thin films successfully deposited on silicon substrate have been studied and discussed. SEM images show the formation of local black circles and hexagonal shapes probably due to the nucleation of a new Si-gel component or phase induced by annealing. Micro Xray Fluorescence mapping shows that the high values of $\mathrm{Si}$ and $\mathrm{B}$ atoms ( $\cong 7$ and 4 normalized unit respectively) can be explained by the fact that the substrate is p-type silicon. The most interesting result of optical measurements is the very good absorption for all the thin films in UV, Visible and NIR regions with values from $70 \%$ to $90 \%$ in UV, from $75 \%$ to $93 \%$ in Visible and NIR. Tauc plots present particularities (rarely encountered behavior) with different segments or absorption changes showing the presence of multiple band gaps coming from the heterogeneity of the thin films (nanowires, gel and nanoparticles). Their values are 1.9 and 2.8 $\mathrm{eV}$ for DKRN-Gel, 2.1 and $3.1 \mathrm{eV}$ for DKRN-UD and 2.1 and $3.2 \mathrm{eV}$ for DKRN-D) corresponding respectively to the band gap of nanowires and that of the gel while the last ones correspond to the undoped and doped nanoparticles (3.1 and $3.2 \mathrm{eV}$ respectively).
\end{abstract}

\section{Keywords}

Nanostructures, Perovskite Nanoparticles, Silicon, Thin Layer, Band Gap 


\section{Introduction}

Among various everlasting desires of all the materials scientists, the most important is the search of materials with as maximum functionalities as possible [1] [2]. The transition metals have very rich chemistry due to variable oxidation state, which offer multiple properties, if selected judicially. A huge number of metal oxides has been investigated and is being studied for various technological applications [3] [4] [5] [6]. As the global concern for the financial and environmental costs of traditional energy resources increases, research on renewable energy, most notably solar energy, has taken center stage. Many alternative photovoltaic (PV) technologies for "the next generation solar cell" have been extensively studied. The novel photovoltaic mechanism for the next generation solar cells using silicon nanostructures and ferroelectric nanoparticles were investigated. Studies by Fridkin et al. [7] [8] demonstrated that the illumination of ferroelectric inorganic materials leads to the creation of a photocurrent. Current-voltage characteristics under lithium niobate $\left(\mathrm{LiNbO}_{3}\right)$ [7] [9] [10] [11] [12] [13] or lead oxides [14] [15] demonstrate that these materials generate a photocurrent, although this one is weak (of the order of $\mathrm{nA} / \mathrm{cm}^{2}$ ), due to the low conductivity of these materials. The low band gap of the bismuth ferrite $\left(\mathrm{BiFeO}_{3}\right)(2.7 \mathrm{eV}$ [16] [17]), compared to other ferroelectrics $\left(\mathrm{LiNbO}_{3}, \mathrm{BaTiO}_{3}\right)$ or lead zirconate titanate ceramics $\mathrm{Pb}\left(\mathrm{Zr}_{\mathrm{x}} \mathrm{Ti}_{1-\mathrm{x}}\right) \mathrm{O}_{3}$ called PZT have made him a better candidate in this field. Kim and al. have studied the influence of the insertion of a ferroelectric film in inorganic photovoltaic cells [18]. Ferroelectric lead compounds having a perovskite structure, such as $\mathrm{Pb}\left(\mathrm{Zn}_{1 / 3} \mathrm{Nb}_{2 / 3}\right) \mathrm{O}_{3}$ ( $\mathrm{PZN}$ ) [19], $\mathrm{Pb}\left(\mathrm{Mg}_{1 / 3} \mathrm{Nb}_{2 / 3}\right) \mathrm{O}_{3}$ (PMN) [20], and their solid solutions with $\mathrm{Pb}\left(\mathrm{TiO}_{3}\right)$ (PT), have been investigated for high-performance ultrasonic transducer applications [21] [22]. Indeed, these last years, high levels of piezoelectricity have been published for $\mathrm{Pb}\left(\mathrm{Mg}_{1 / 3} \mathrm{Nb}_{2 / 3}\right) \mathrm{O}_{3}-\mathrm{PbTiO}_{3}$ (PMN-PT) and PZN-PT single crystals: d33 > 2500 $\mathrm{pC} / \mathrm{N}, \mathrm{d} 31>-1500 \mathrm{pC} / \mathrm{N}, \mathrm{k} 33>90 \%, \mathrm{k} 31>80 \%$ and $\mathrm{kt}>60 \%$ [23] [24] [25] [26], showing at least ferroelectric, ferroelastic and piezoelectric properties. However, the greatest difficulty to use such single crystals on electronic devices is to achieve them in thin layers form because of their incongruent melting property. The integration of such materials as thin films has attracted considerable research attention thanks to their outstanding performances that allow to consider new features for the realization of photovoltaic device.

In this paper, to integrate them into silicon nanostructures, we realized PZNPT nanoparticles deposition, already synthesized by the so-called solution flux method [25], as thin film on p-type nanoporous silicon substrate. Structural, morphological, optical and electrical properties of such nanoparticles and their as deposited thin film were investigated.

\section{Experimental Procedure}

A boron doped p-type (100) silicon wafer of $600-650 \mu \mathrm{m}$ of thickness with resistivity of $1-5 \Omega \cdot \mathrm{cm}$ of the one polished face was purchased from BT Electronics. 
Silver assisted chemical etching method was used for nanowires fabrication as described elsewhere [27]. Samples, after cleaning, are immersed in the etching solution containing $\mathrm{HF}(22.8 \mathrm{M}) / \mathrm{Ag}\left(\mathrm{NO}_{3}\right)_{2}(0.02 \mathrm{M})$ mixture in a volume ratio of $\mathrm{v}: \mathrm{v}=6: 19$ for a preselected time. The samples were placed directly, after rinsed with deionized water, in the $\mathrm{HF}(40 \%) / \mathrm{H}_{2} \mathrm{O}_{2}$ solution in volume ratio of $\mathrm{v}: \mathrm{v}=20: 4$. The sample was subsequently immersed in a $\mathrm{HNO}_{3}$ solution $(0.1$ $\mathrm{M}) / \mathrm{H}_{2} \mathrm{O}$ in a volume ratio of $\mathrm{v}: \mathrm{v}=23: 10$. Finally the samples were immersed for at least 8 hours [8] in an $\mathrm{HNO}_{3} / \mathrm{HCl} / \mathrm{H}_{2} \mathrm{O}$ solution (v:v:v = 1:1:1) to remove the etchant excess. Undoped and Mn doped PZN-4.5PT single crystals synthesized by the flux method [25] were grounded in a mortar to obtain a very fine powder (TEM characterizations revealed nanoparticles of the nanometer order and studies that are not shown here have confirmed that the population of spherical particles having a diameter of $30 \mathrm{~nm}$ was more representative).

\subsection{PZN-PT Nanoparticles Thin Film Fabrication}

The grounded powder was dispersed in a gel fabricated in the laboratory LCPM in Assane Seck University of Ziguinchor. To obtain a homogeneous film, spin coating process was carried out at room temperature using a spin coater Midas $1200 \mathrm{D}$ model at $3500 \mathrm{rpm}$ with an initial acceleration of 5 seconds and an operating time of $10 \mathrm{~min}$. After $10 \mathrm{~min}$ bake in oven at $100^{\circ} \mathrm{C}$ (at $100^{\circ} \mathrm{C}$ there is good adhesion of the deposited thin layer as shown by D. Kobor et al.), thermal annealing in a K114 type muffle furnace was performed. Heating was carried out at $900^{\circ} \mathrm{C}$ for the gel diffusion through the p-type silicon nanowires with a heating rate between 10 and $20^{\circ} \mathrm{C} / \mathrm{min}$ and $30-60 \mathrm{~min}$ plateau. Different samples were fabricated using the two types of nanopowders (undoped PZN-4.5PT powder and $1 \% \mathrm{Mn}$ PZN-4.5PT doped powder) and characterized.

\subsection{Surface Characterization}

The as-synthesized powders were characterized by TEM analysis using a FEI CM10 microscope (Philips) by depositing a drop of nanoparticles suspension on carbon coated copper grids placed on a filter paper. Surface morphology and composition of thin films samples were observed respectively by using a MERLIN FEG of Zeiss scanning electron microscopy model in ICMPE (ParisEst) and an electron beam lithography system Pioneer Raith model in $\mathrm{C}(\mathrm{PN}) 2$ (Paris 13 University). To measure the thickness and quantify surface roughness we used a Bruker profilometer Dektak XT model. The data are processed with Vision 64 software. Atoms such as Si, B and P distribution on thin layer surface is performed using Micro Xray Fluorescence mapping with beamline ID12 at the European Synchrotron Radiation Facility (ESRF) in Grenoble, France.

\subsection{Optical Properties}

Optical properties of thin films were investigated using an UV-Visible spectrophotometer with an integrating sphere model LAMBDA 950S in IM2NP (Marseille). 
Measurements are made with a pitch of 5 on a wavelength spectrum ranging from 250 and $1500 \mathrm{~nm}$.

\section{Results and Discussion}

\subsection{TEM and SEM Images}

Figure 1 presents TEM micrographs of the undoped PZN-4.5PT (Figure 1(a)) and $1 \% \mathrm{Mn}$ doped PZN-4.5PT (Figure 1(b) and Figure 1(c)) nanoparticles. According to these images, the undoped crystals consisted mainly of spherical nanoparticles with a diameter between 30 and $100 \mathrm{~nm}$, whereas the doped ones were made up of both spherical nanoparticles with diameters ranging from 30 to $100 \mathrm{~nm}$ (Figure 1(b)) and nanosticks with lengths up to $200 \mathrm{~nm}$ and diameters smaller than $10 \mathrm{~nm}$ (Figure 1(c)).

Figure 2 shows the SEM images for different materials thin layers. The images of Figure 2(a) and Figure 2(b) (increasing magnification) confirm surface nanostructuration with MACE method showing porous silicon nanowires. Figure 2(c) and Figure 2(d) images reveal that the gel is diffused inside the nanowires and covered totally the whole surface. From Figure 2(e) and Figure 2(g) a homogeneous distribution of undoped and 1\% Mn doped PZN-4.5PT nanoparticles can be seen. However, it is interesting to notice a local black circles formation (Figures 2(e)-(g)) characterized by circles and hexagonal shapes (Figure $2(\mathrm{e})$ and Figure $2(\mathrm{~h})$ ). This is probably due to the formation of a new Si-gel component or phase induced by annealing. The black films observed on Figure 2 (e) and Figure 2(g) would come from the new component (Si-gel) thin layer coalescence.

Figure 3 shows the mapping of $\mathrm{Si}, \mathrm{P}$ and $\mathrm{B}$ atoms distribution in the gel film deposited on silicon nanowires. The color scale is given in counts per seconds.

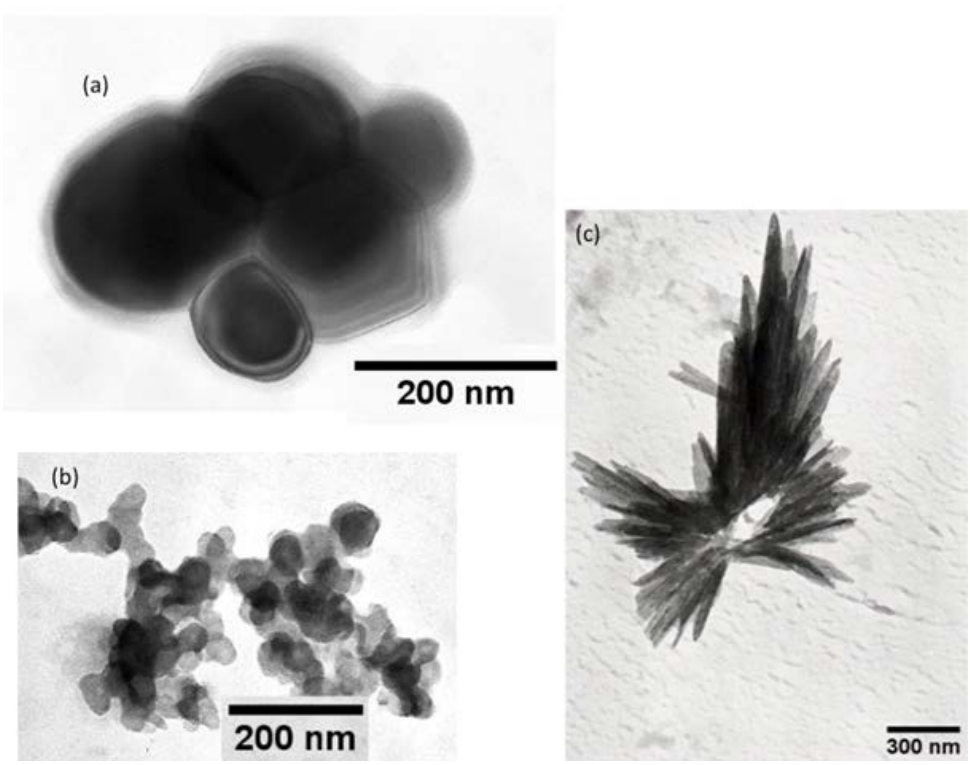

Figure 1. TEM images of (a) undoped PZN-4.5PT, (b) spherical and (c) sticks shapes of $1 \%$ Mn doped PZN-4.5PT nanoparticles. 

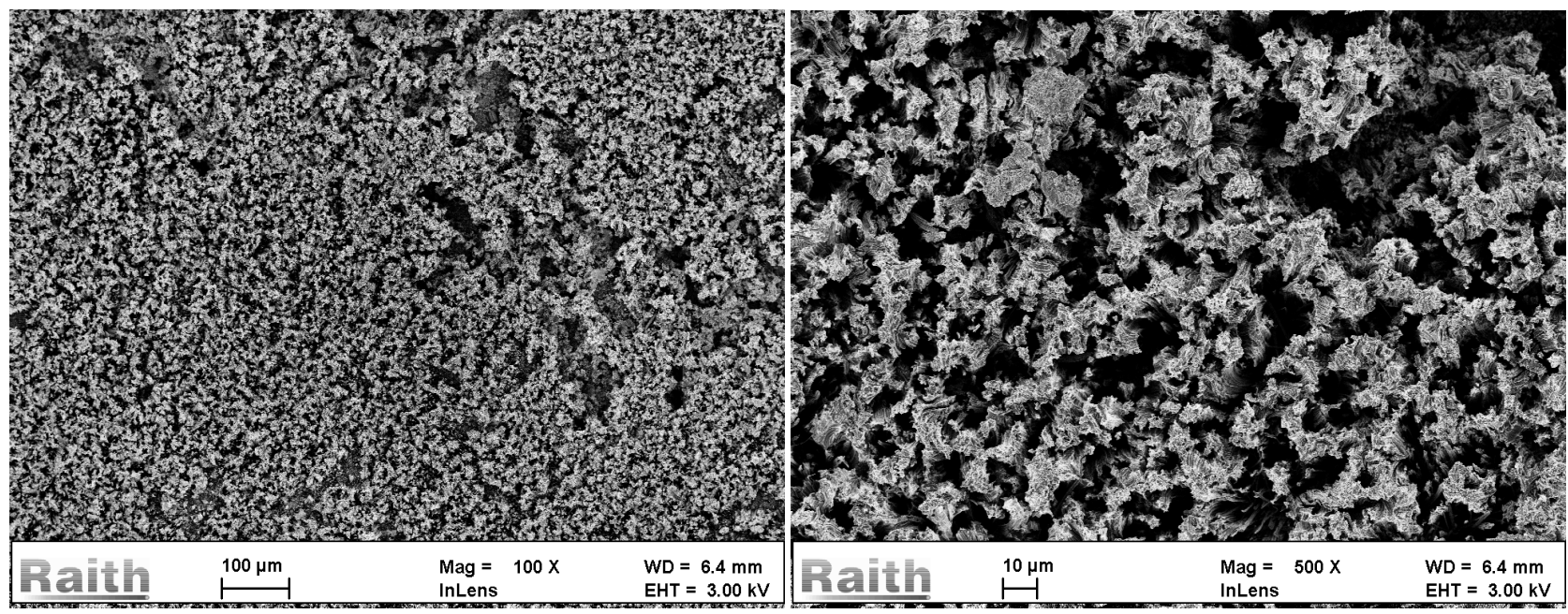

(a)

(b)

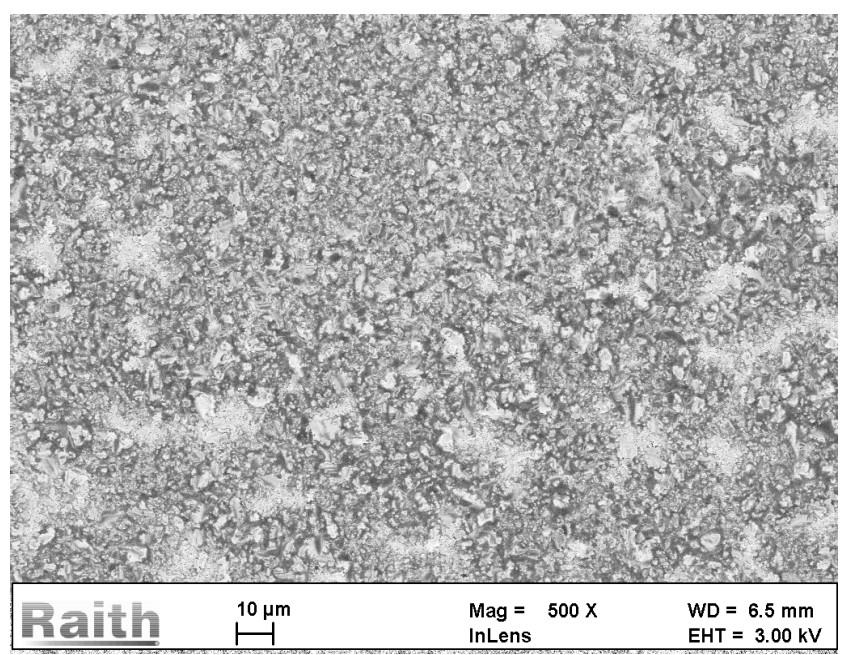

(c)

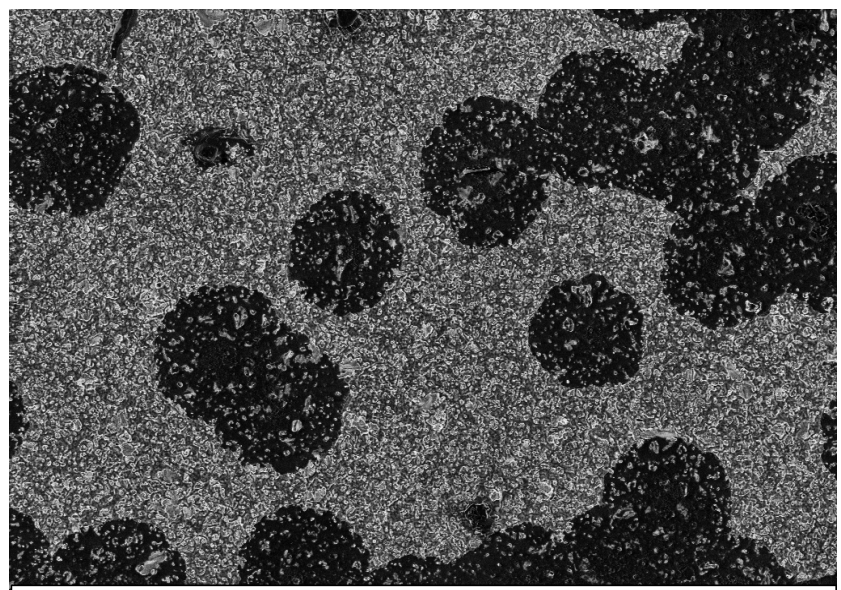

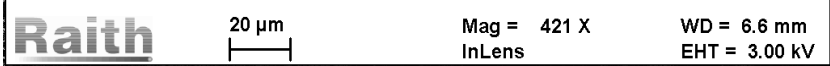

(e)

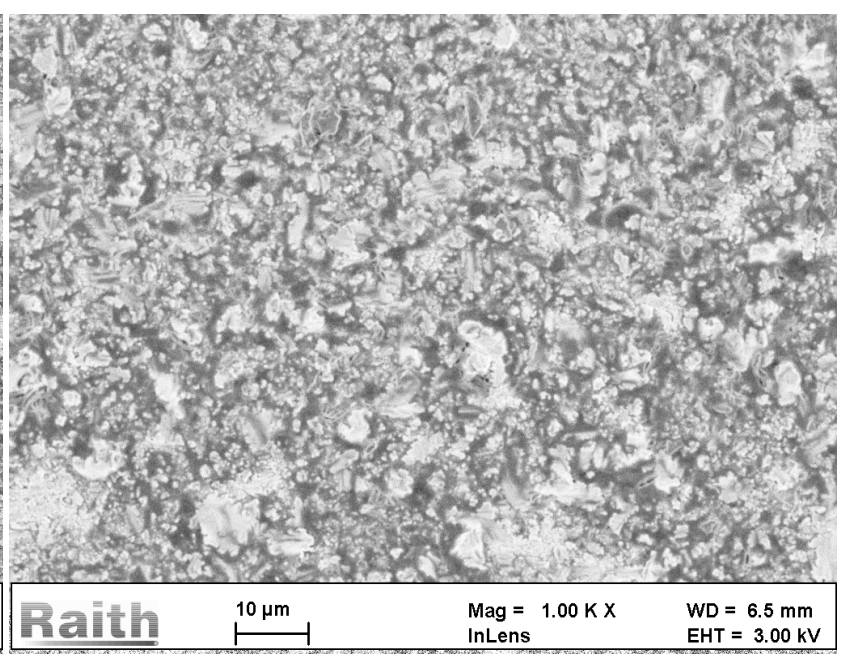

(d)

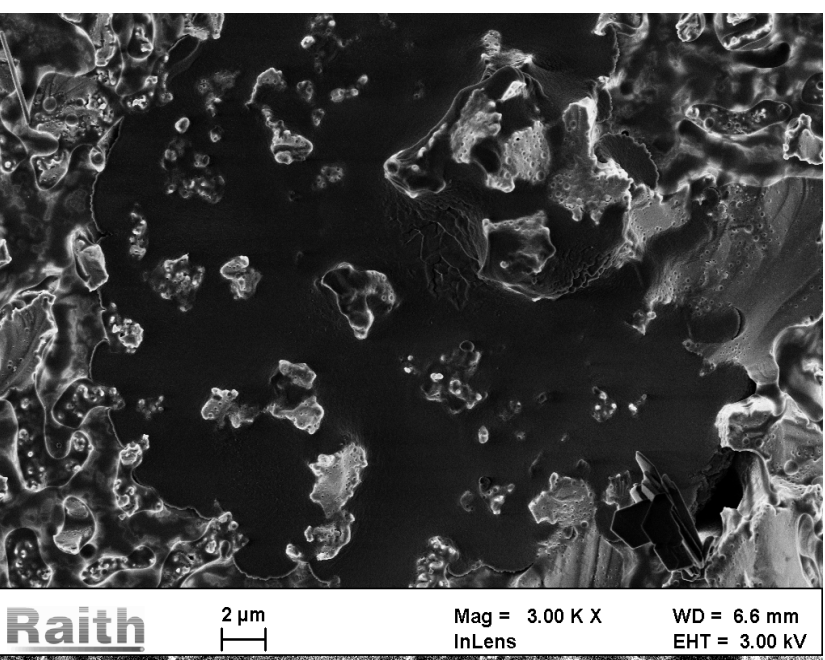

(f) 


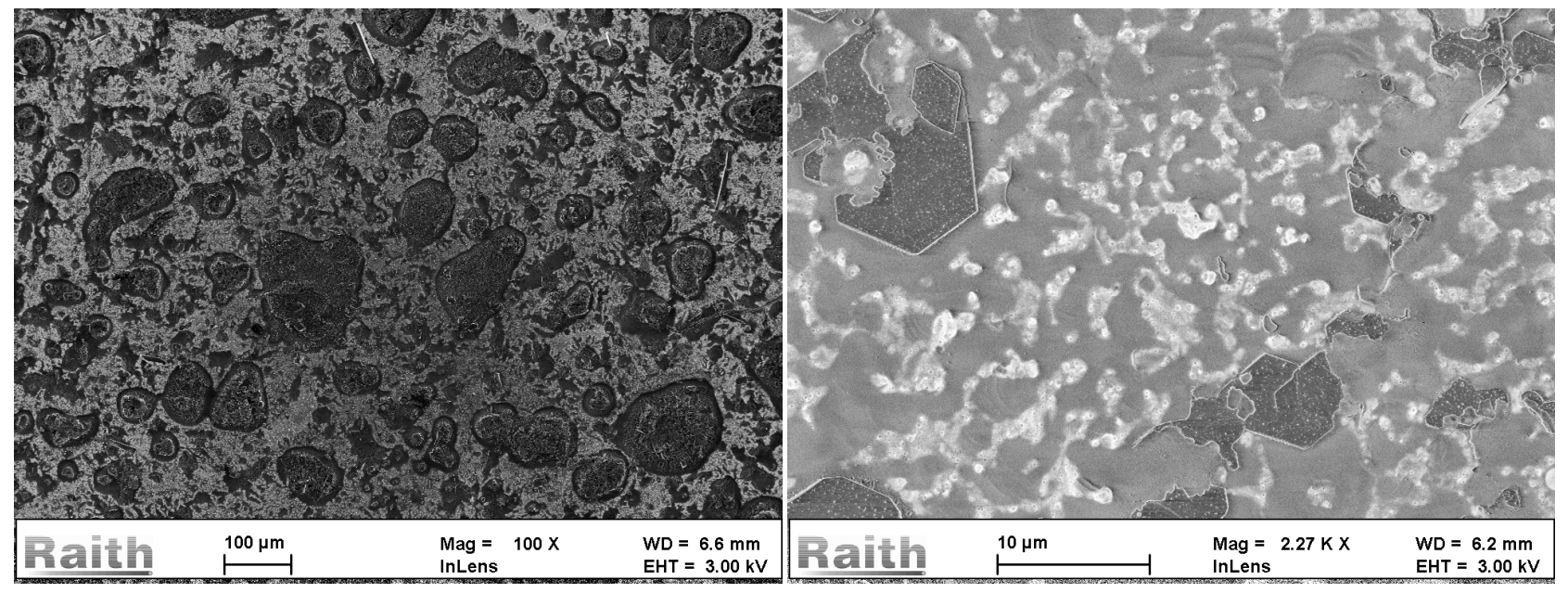

Figure 2. SEM images of (a) and (b) p type Si-nanowires, (c) and (d) gel deposited thin film on Si nanowires, (e) and (f) undoped and $(\mathrm{g})$ and $(\mathrm{h}) 1 \% \mathrm{Mn}$ doped PZN-4.5PT dispersed in gel deposited films on Si nanowires.
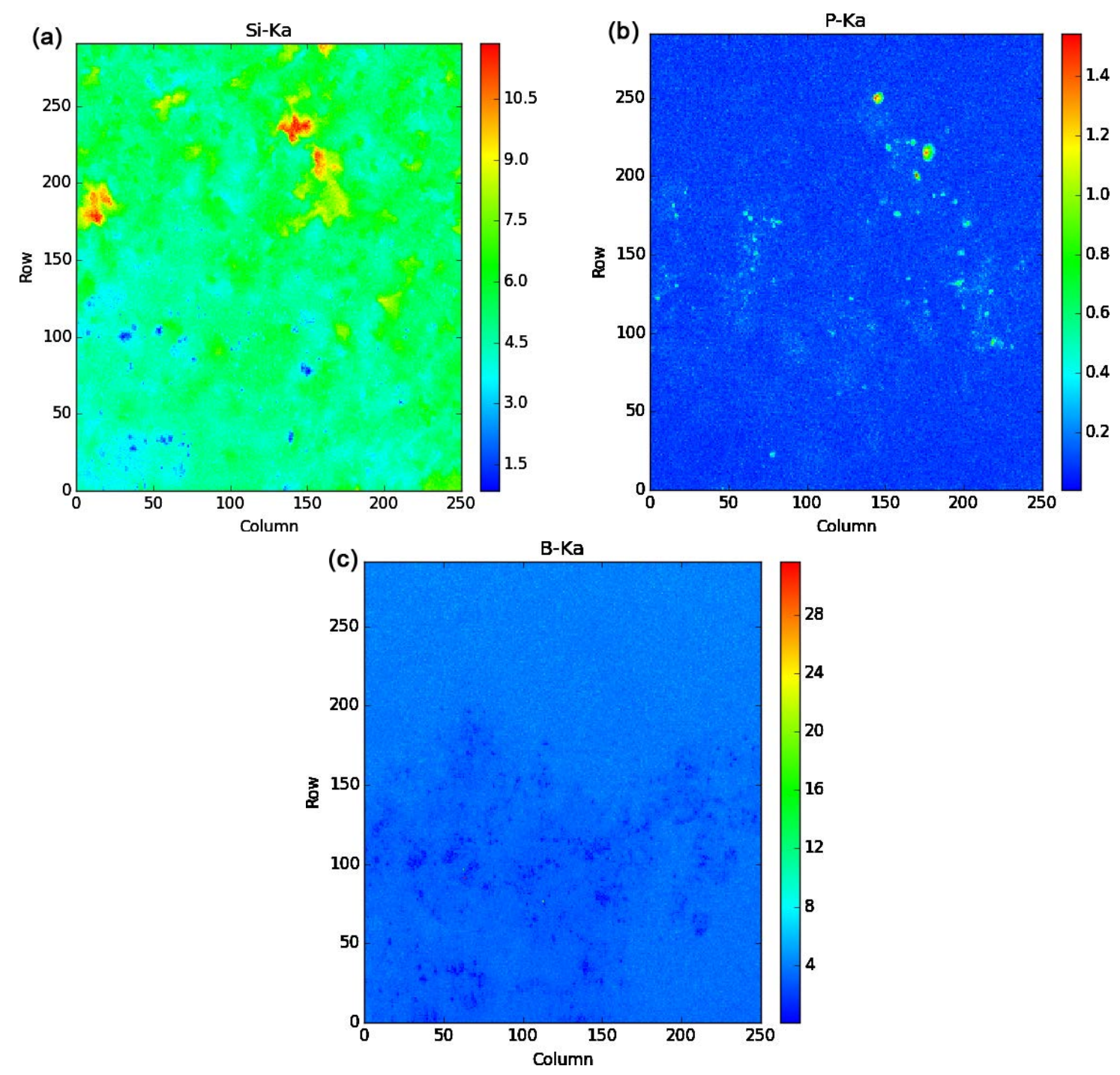

Figure 3. Micro X-ray fluorescence mapping of (a) Si atom, (b) P atom, (c) B atom distribution on the DKRN-Gel sample. 
The signal intensity in Figure 3(a) shows the high content of Si compared to B and $\mathrm{P}$, which represent the substrate dopants. The ratio between the dopants and silicon intensities is in agreement with these elements doping concentration (1016 at $/ \mathrm{cm}^{3}$ for B and $1019 \mathrm{at} / \mathrm{cm}^{3}$ for P).

These high values of $\mathrm{Si}$ and $\mathrm{B}$ (around 7 and 4 counts/s respectively) can be explained by the fact that it is a p-type silicon substrate (Figure 3(a) and Figure $3(\mathrm{c})$ ). The low value of phosphorus concentration (around $0.2 \mathrm{count} / \mathrm{s}$ ) compared to boron is surprisingly, however this could be explained by the possibility of $\mathrm{P}$ atoms to be diffused more deeply inside nanowires and the bottom of these latters than on their top surface (Figure 3(b)) creating less concentration at the layer surface.

Figure 4 shows the mapping $(1 \mathrm{~mm} \times 1 \mathrm{~mm})$ obtained with the mechanical

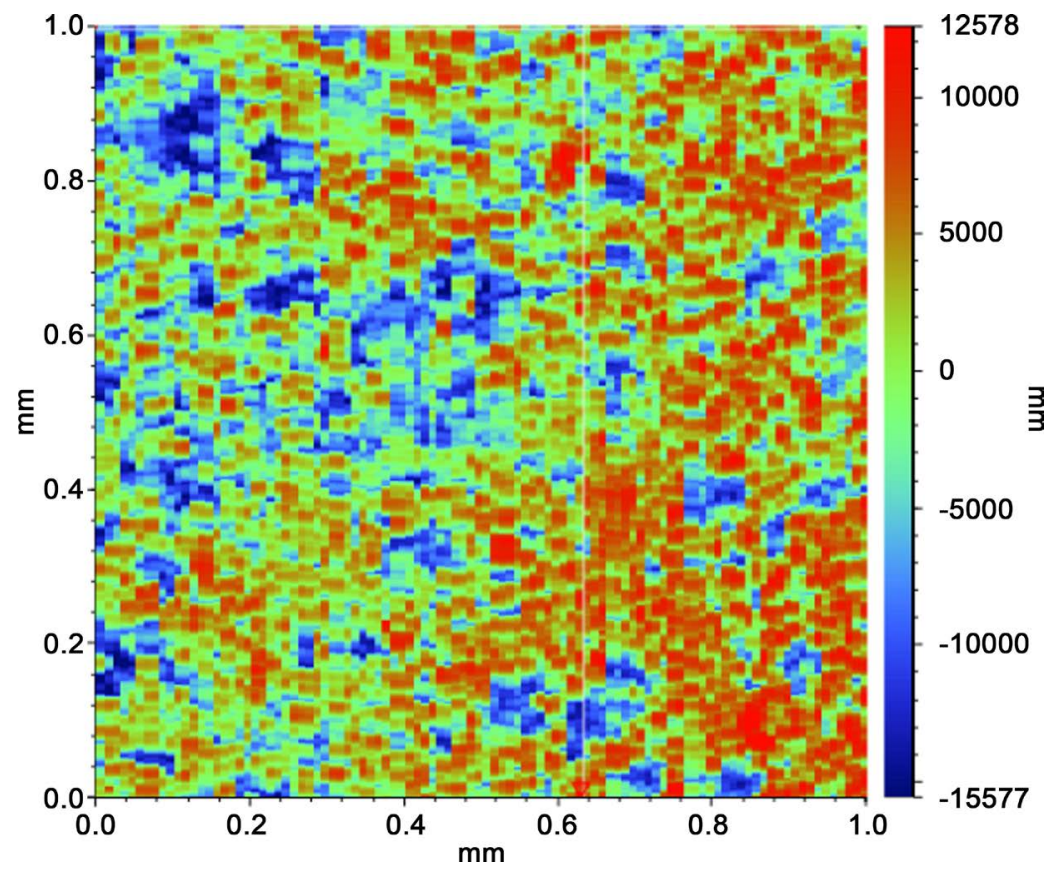

(a)

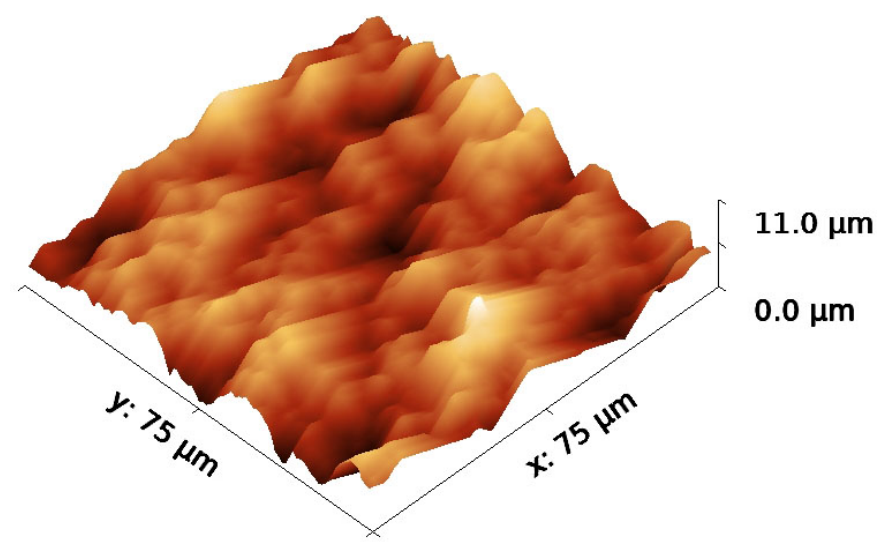

(b)

Figure 4. Roughness mapping of silicon nanowires DKRN-NW surface by (a) mechanical profilometer and (b) atomic force microscopy. 
profilometer Dektak XT model (Figure 4(a)) and the image of the atomic force microscopy (Figure 4(b)). The root mean square roughness (RMS) measured by the mechanical profilometer and the atomic force microscopy is $1.4 \mu \mathrm{m}$. We have a high roughness, which causes the phenomenon of diffusion of the incident light which results in a decrease in transmittance in infrared region [28].

\subsection{Optical Properties}

\subsubsection{Reflectance and Absorbance}

Figure 5 shows UV-Visible reflectance and absorbance curves for Si p-type

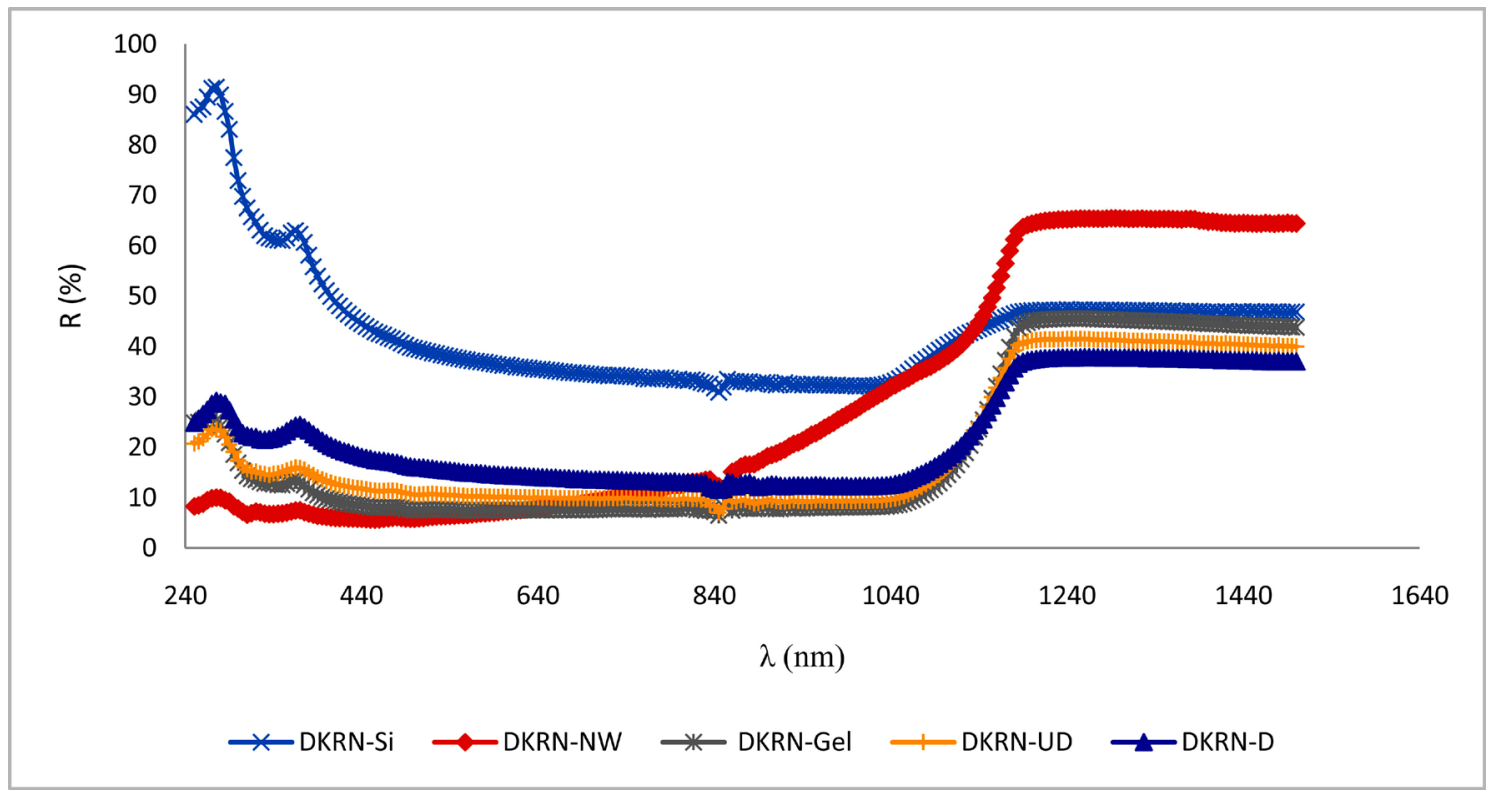

(a)

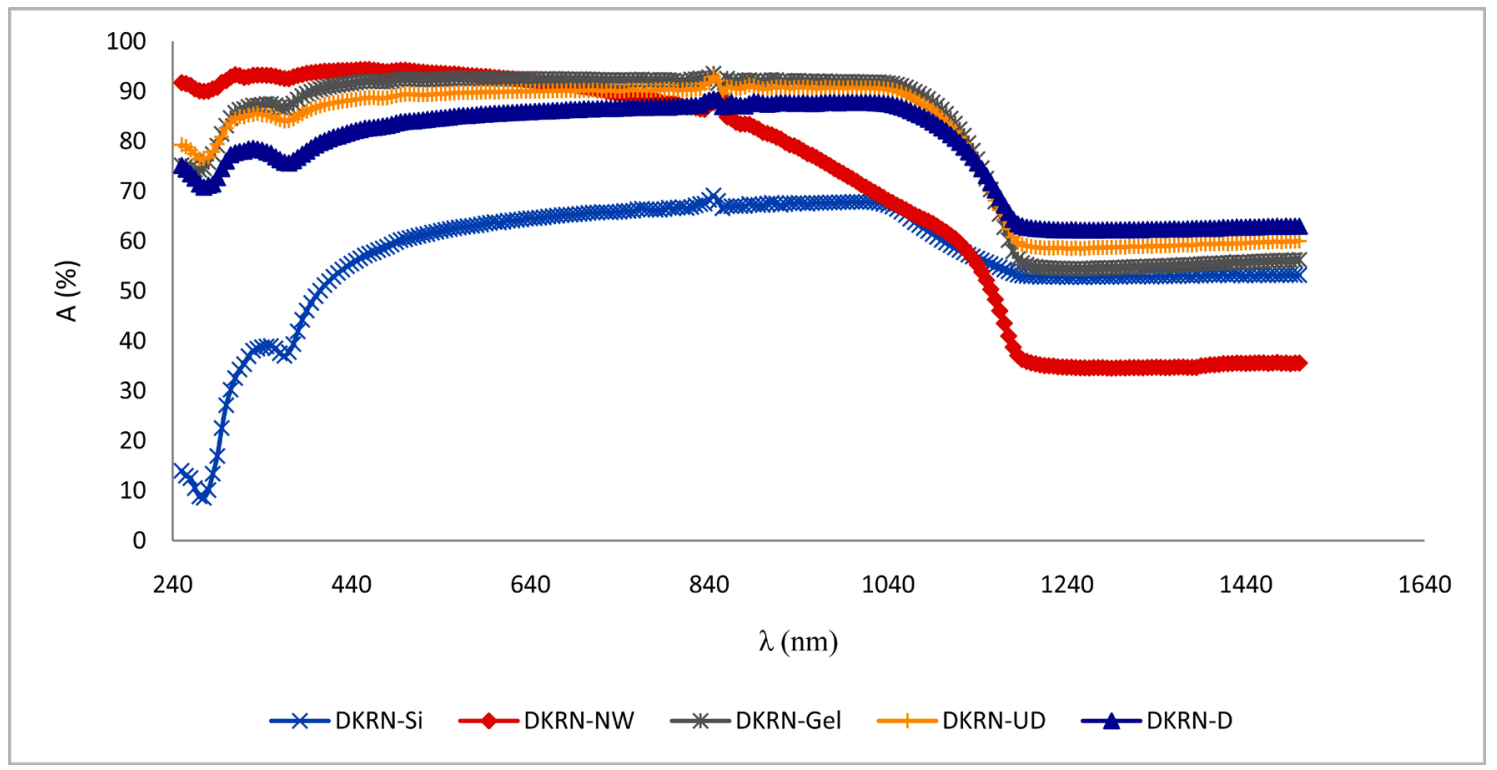

(b)

Figure 5. (a) Reflectance and (b) absorbance according to the UV-Visible-NIR wavelength for different fabricated samples. 
substrate (DKRN-Si), p-type Si nanowires (DKRN-NW), gel deposited on p-type Si nanowires surface (DKRN-Gel), undoped (DKRN-UD) and 1\% Mn doped (DKRN-D) PZN-4.5PT nanoparticles dispersed in the gel and deposited on the p-type Si nanowires. The nanostructured surface (nanowires) has a significant influence on the reflectance value and is in agreement with the literature (5\% 15\%) [29]. It decreases from over $45 \%$ for the p-type Si substrate in the wavelength range of 250 to $1500 \mathrm{~nm}$ to less than $10 \%$ for the nanostructured silicon. For DKRN-UD and DKRN-D, reflectance decreases from 45 to less than 20\% (Figure 5(a)). However, their values increased compared to DKRN-Gel, which has the lowest reflectance value (around 10\%) in the whole measurement range. This behavior of the thin film from perovskite nanoparticles could be explained by the high reflectance values of PZN-PT single crystals (20\% - 25\%) [30]. The absorbance varies reversely to the reflectance and as expected DKRN-NW sample shows a very high absorption in UV region (90\%) before dropping down in visible and near IR region (Figure 5(b)). DKRN-Gel and DKRN-UD samples exhibited very interesting highest values and stable behavior in Visible and NIR region (90\% - 93\%), which decrease slightly in UV region below DKRN-NW values. DKRN-D sample absorbance has the same behavior as DKRN-UD with decreasing values. The most interesting result is the very good absorption for all the thin films in UV, Visible and NIR regions with values from $70 \%$ to $90 \%$ in UV, from $75 \%$ to $93 \%$ in Visible and NIR. These results allow achieving good absorption materials over the entire useful wavelength, for photovoltaic conversion efficiency improvement. The good absorption of DKRN-Gel justifies its choice to dissolve undoped and $1 \%$ Mn doped nanoparticles.

\subsubsection{Determination of Refractive Index and Band Gap}

The values of the refractive indices are determined from the reflection spectra of Figure 5 using the following equation [31]:

$$
R_{\min }=\left(\frac{n_{1}^{2}-n_{0} n_{2}}{n_{1}^{2}+n_{0} n_{2}}\right)^{2}
$$

where $R_{\min }$ is the minima taken from the spectrum, $n_{1}$ is the reflective index of the layer, $n_{0}\left(n_{0}=1\right)$ and $n_{2}\left(n_{2}=3.4777\right)$ are respectively the refractive index of air and Silicon substrate. The layer's refractive index $n_{1}$ was determined from the following relation:

$$
n_{1}=\left(\frac{n_{0} n_{2}\left(1+\sqrt{R_{\min }}\right)}{1-\sqrt{R_{\min }}}\right)^{1 / 2}
$$

From reflection spectra of Figure 6(a) and applying the relations (1) and (2), we obtain the refractive index. Their values are 2.37, 2.40, 2.44 and 2.60 respectively for DKRN-NW, DKRN-Gel, DKRN-UD, and DKRN-D thin layers (Table 1). These values confirm their possible use as antireflective and passivation thin layers for solar cells efficiency improvement. The values are slightly equal to those found for antireflective and passivation materials for silicon photovoltaic 


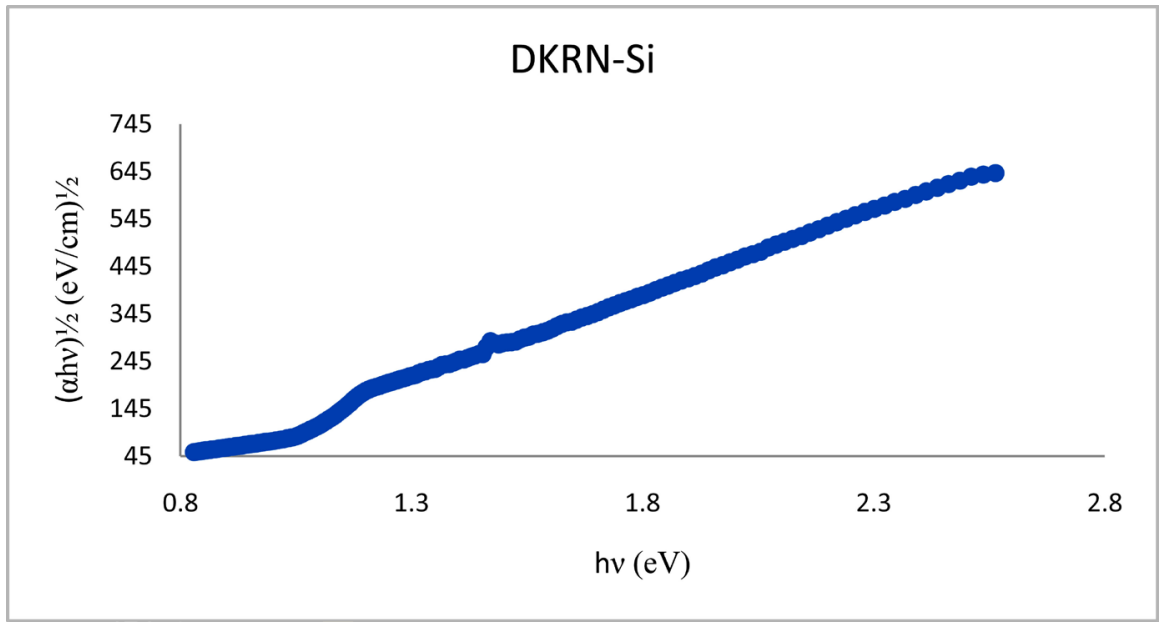

(a)

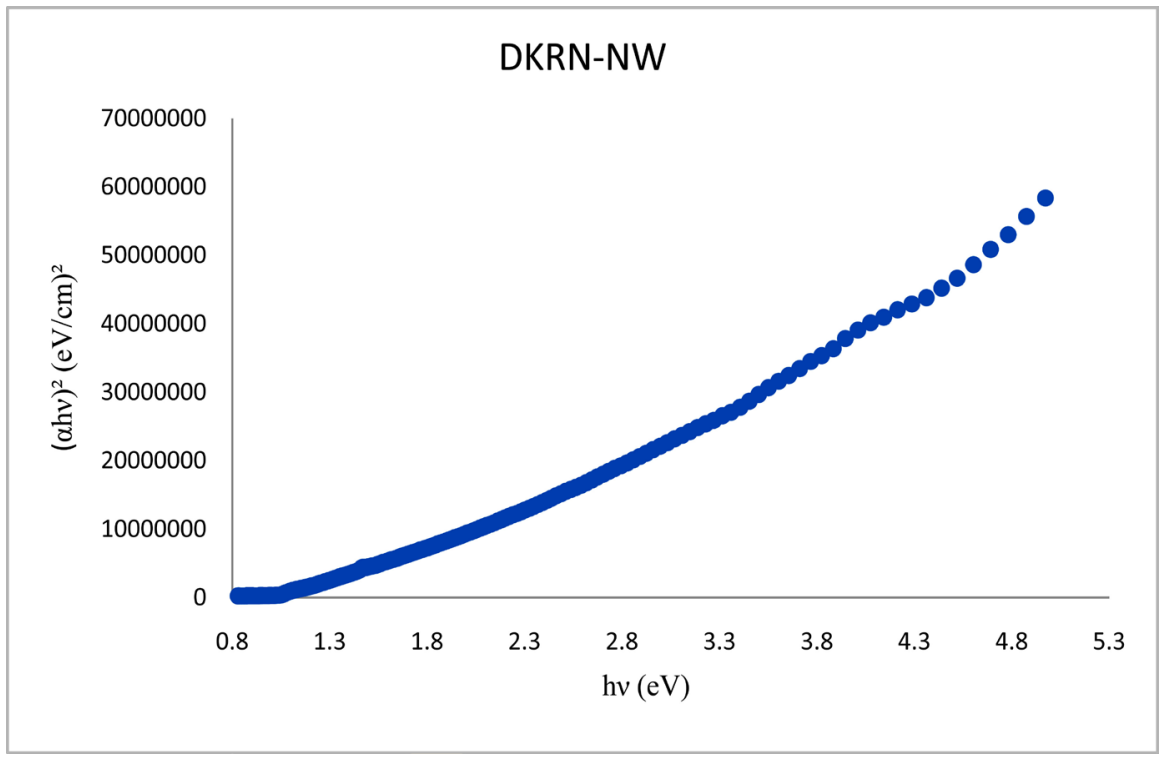

(b)

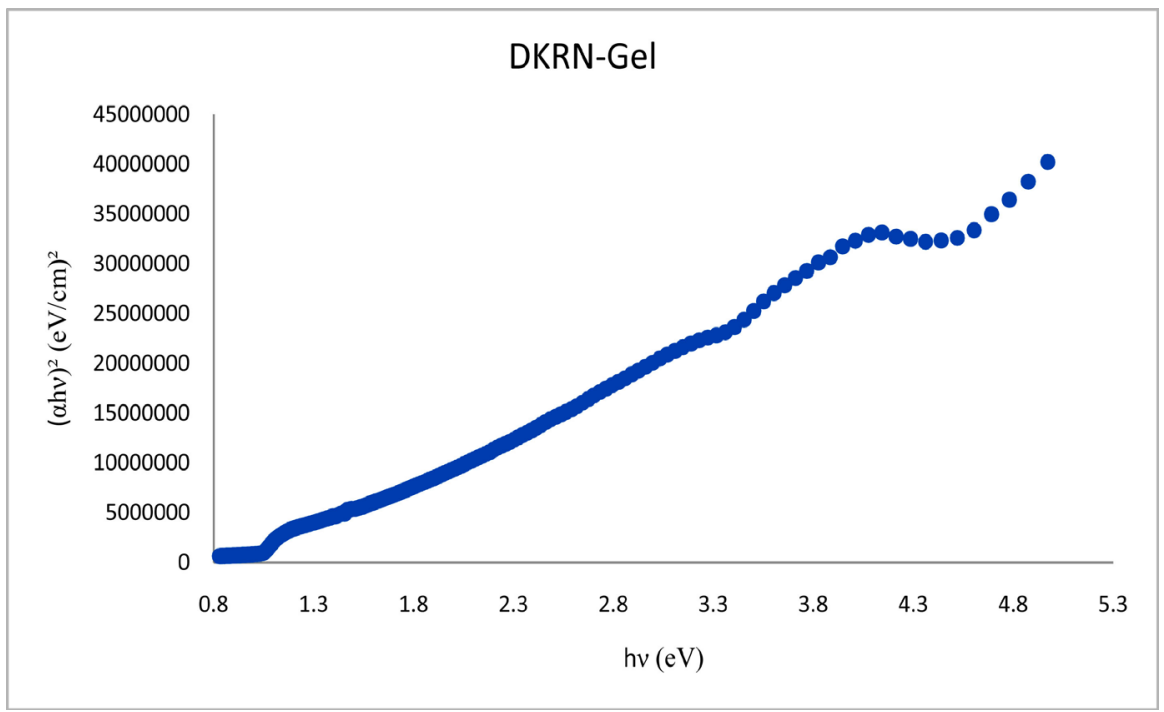

(c) 


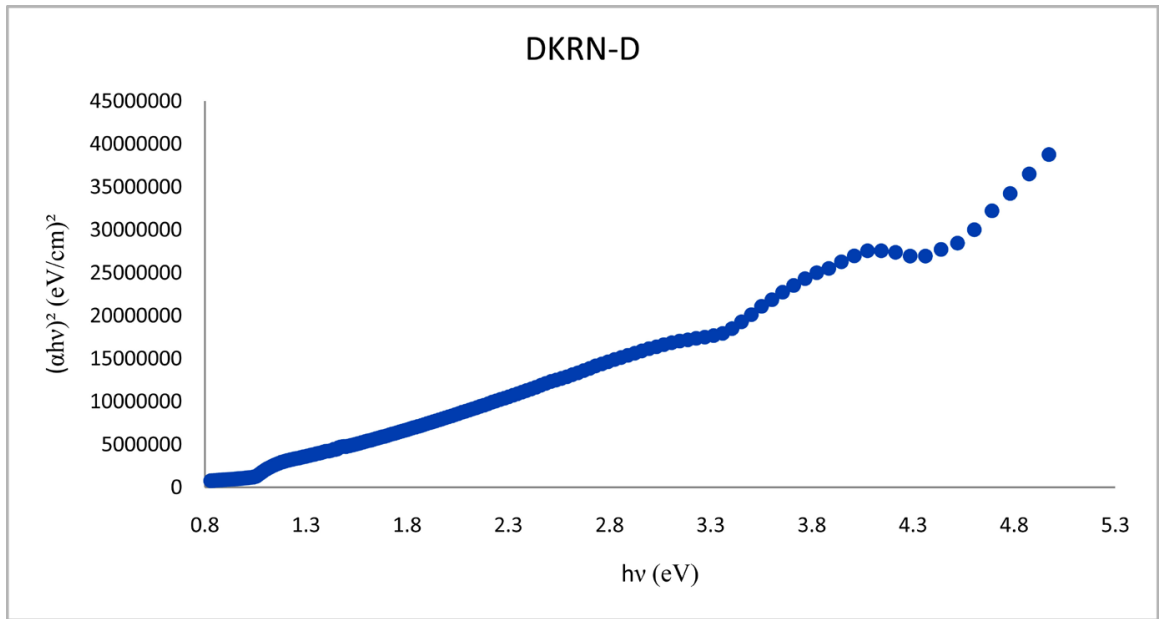

(d)

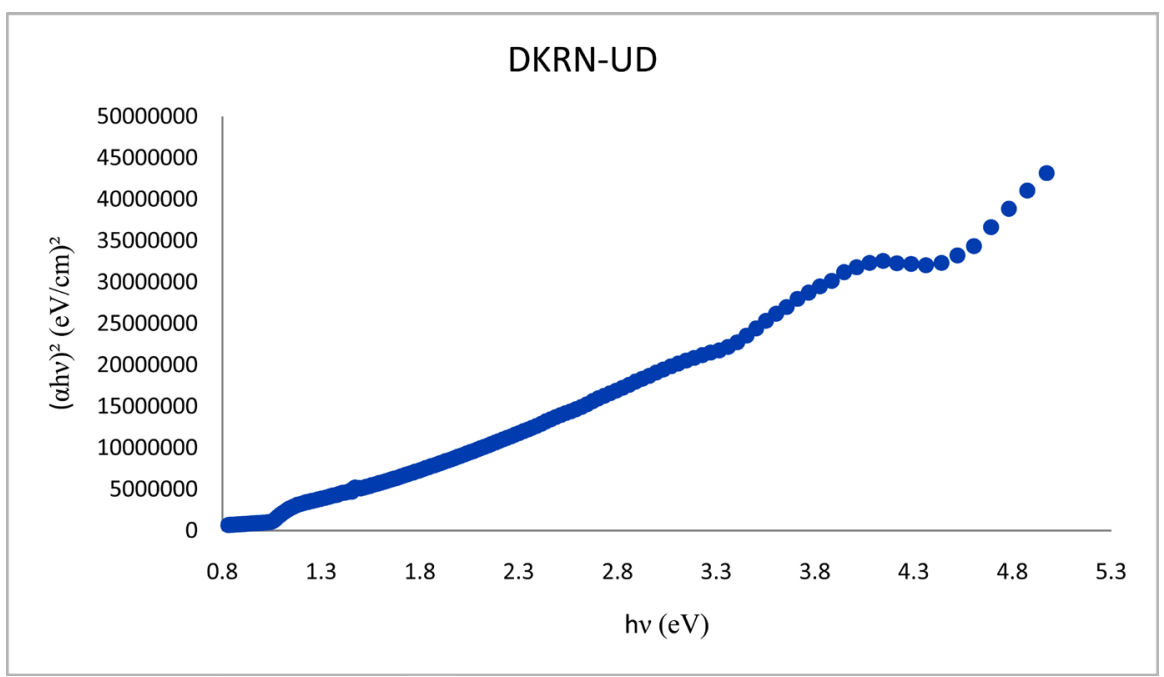

(e)

Figure 6. Tauc plot curves of (a) DKRN-Si, (b) DKRN-NW, (c) DKRN-Gel, (d) DKRN-D, (e) DKRN-UD samples.

Table 1. Refractive index and optical gap values.

\begin{tabular}{cccc}
\hline Samples & $\mathrm{n}$ & Eg1 $(\mathrm{eV})$ & Eg2 (eV) \\
\hline DKRN-Si & 3.47 & - & 1.05 \\
DKRN-NW & 2.37 & - & 2.50 \\
DKRN-Gel & 2.40 & 1.90 & 2.80 \\
DKRN-UD & 2.44 & 2.10 & 3.10 \\
DKRN-D & 2.60 & 2.10 & 3.20 \\
\hline
\end{tabular}

\section{(2.00 for $\mathrm{ZnO}, 2.04$ for Si3N4).}

Figure 6 shows Tauc plots of the samples considering them as direct gap transitions. Optical gap are determined using Tauc relation [32]:

$$
(\alpha h v)=A\left(h v-E_{g}\right)^{n}
$$


where $A$ is constant and $h v$ is the photon energy, $\alpha$ is the absorption coefficient.

The different values are summarized in Table 1.

The figures present particularities, rarely encountered behavior, with different segments or absorption changes showing the presence of multiple band gaps coming from the heterogeneity of the thin films (nanowires, gel and nanoparticles).

The Si band gap gets transformed from an indirect into a pseudo-direct one resulting in photoluminescence (PL) and electroluminescence (EL) of the Silicon nanowires as a consequence of quantum confinement effects [33]. The quantum confinement clearly led to a widening of the gap. In these nanostructures, small by definition, the charges remain close to each other. The recombination probability is increased and the radiative lifetime decreases and then increases the radiative efficiency. Furthermore the Heisenberg uncertainty imposes, since the position of charges is well known, an expansion of the waves function in the $\mathrm{k}$ space. Thus the transition could be done and constant $\mathrm{k}$ would induce a change in the gap type of our materials from indirect to the pseudo-direct one [34]. Silicon energy band gap is confirmed by the Figure 5(a), which is equal to $1.05 \mathrm{eV}$ in good agreement with literature $(1.12 \mathrm{eV})$. Tauc plots of DKRN-NW, DKRNGel, DKRN-UD and DKRN-D (Figures 6(b)-(e)) present different absorption zones that could be due to the presence of at least two band gaps. We then found one value $(2.5 \mathrm{eV})$ for the nanowires on $\mathrm{Si}$ substrate while in the deposited thin films we found two energy values (1.9 and $2.8 \mathrm{eV}$ for DKRN-Gel, 2.1 and $3.1 \mathrm{eV}$ for DKRN-UD and 2.1 and $3.2 \mathrm{eV}$ for DKRN-D) corresponding respectively to the band gap of nanowires and that of the gel. The last ones are considered to be from nanowires $(2.1 \mathrm{eV})$ and the undoped $(3.1 \mathrm{eV})$ and $\mathrm{Mn}$ doped $(3.2 \mathrm{eV})$ nanoparticles thin layers. These increases can be explained by the high value of the band gap for the PZN-PT materials around to $3.04 \mathrm{eV}$ [35] for the nanoparticles. In addition, the presence of the gel and the confinement effects could also explain the increasing for the deposited films compared to the nanowires band gap.

\section{Conclusion}

PZN-4.5PT nanoparticles thin layers were successfully prepared using a gel fabricated in our laboratory. Silicon p-type nanostructured was used to deposit this thin film. SEM images revealed homogeneous distribution of the gel inside the nanowires. Optical properties investigation shows very good absorption for all the thin films in UV, Visible and NIR regions with values from $70 \%$ to $90 \%$ in UV, from $75 \%$ to $93 \%$ in Visible and NIR. Refractive index is measured and shows that their values confirm the possible use of such materials as antireflective and passivation thin layers. The Tauc plots show a behavior rarely encountered in materials like presence of more than one band gaps absorption energy (different changes) confirming the heterogeneity of the thin layers. These results show good optical properties favorable to the solar cell development. 


\section{Acknowledgements}

This work is supported by Agence Universitaire de la Francophonie (AUF).

\section{References}

[1] Benedek, N.A., Mulder, A.T. and Fennie, C.J. (2012) Journal of Solid State Chemistry, 195, 11-20. https://doi.org/10.1016/j.jssc.2012.04.012

[2] Peng, D., Wang, X., Xu, C., Yao, X., Lin, J. and Sun, T. (2013) Journal of the American Ceramic Society, 96, 184-190. https://doi.org/10.1111/jace.12002

[3] Matar, S.F. (2012) Progress in Solid State Chemistry, 40, 31-40. https://doi.org/10.1016/j.progsolidstchem.2012.06.001

[4] Bishop, A.R. (2004) Journal of Physics and Chemistry of Solids, 65, 1449-1454. https://doi.org/10.1016/j.jpcs.2003.07.002

[5] Roesky, H.W., Haiduc, I. and Hosmane, N.S. (2003) Chemical Reviews, 103, 2579-2596. https://doi.org/10.1021/cr020376q

[6] Song, F., Shen, X., Liu, M. and Xiang, J. (2012) Journal of Solid State Chemistry, 185, 31-36. https://doi.org/10.1016/j.jssc.2011.10.009

[7] Fridkin, V.M. and Popov, B.N. (1978) Soviet Physics Uspekhi, 21, 981. https://doi.org/10.1070/PU1978v021n12ABEH005722

[8] Fridkin, V.M. and Popov, B.N. (1978) Ferroelectrics, 21, 611-613. https://doi.org/10.1080/00150197808237344

[9] Glass, A.M., von der Linde, D. and Negran, T.J. (1974) Applied Physics Letters, 25, 233-235. https://doi.org/10.1063/1.1655453

[10] Dalba, G., et al. (1995) Physical Review Letters, 74, 988-991. https://doi.org/10.1103/PhysRevLett.74.988

[11] Garcia-Cabanes, A. and Cabrera, J.M. (1993) Journal of Physics: Condensed Matter, 5, 2267-2276. https://doi.org/10.1088/0953-8984/5/14/022

[12] Simon, M., et al. (1997) Journal of Physics D: Applied Physics, 30, 144. https://doi.org/10.1088/0022-3727/30/1/018

[13] Kang, B., et al. (2006) Optics Communications, 266, 203-206. https://doi.org/10.1016/j.optcom.2006.04.064

[14] Poosanaas, P., et al. (1998) Journal of Applied Physics, 84, 1508-1512. https://doi.org/10.1063/1.368216

[15] Yang, Y.S., et al. (2000) Applied Physics Letters, 76, 774-776. https://doi.org/10.1063/1.125891

[16] Ihlefeld, J.F., et al. (2008) Applied Physics Letters, 92, 142908. https://doi.org/10.1063/1.2901160

[17] Yang, S.Y., et al. (2009) Applied Physics Letters, 95, 062909. https://doi.org/10.1063/1.3204695

[18] Kim, J., Kim, D.-S. and Lee, S.-H. (2003) Solar Cell Using Ferroelectric Material. US6639143.

[19] Bokov, V.A. and Myl'nikova, I.E. (1960) Soviet Physics Solid State, 2, 2428.

[20] Smolenskii, G.A., Isupov, V.A., Agranovskaya, A.I. and Popov, S.N. (1961) Soviet Physics Solid State, 2, 2584.

[21] Nomura, S., Takahashi, T. and Yokomizo, Y. (1969) Journal of the Physical Society of Japan, 27, 262. https://doi.org/10.1143/JPSJ.27.262 
[22] Mulvihill, M.L., Park, S.E., Risch, G., Li, Z., Uchino, K. and Shrout, T.R. (1996) The Japanese Journal of Applied Physics, 35, 3984. https://doi.org/10.1143/JJAP.35.3984

[23] Lebrun, L., Zhang, S., Randall, C.A., Shrout, T.R. and Guyomar, D. (2002) Ceramic Transactions, 136, 117.

[24] Benayad, A., Kobor, D., Lebrun, L., Guiffard, B. and Guyomar, D. (2004) Journal of Crystal Growth, 270, 137-144. https://doi.org/10.1016/j.jcrysgro.2004.06.017

[25] Dupret, F. and Van Den Bogaert, N. (1994) Growth Mechanisms and Dynamics, Part B. In: Hurle, D.T.J., Ed., Handbook of Crystal Growth, Vol. 2, North-Holland, New York.

[26] Prya, S. and Uchino, K. (2002) Journal of Applied Physics, 91, 4515. https://doi.org/10.1063/1.1459101

[27] Touré, M., Kobor, D., Ndiaye, L.G., Ndiaye, A., Tine, M., Bastide, S., Thaury, M.A.P. and Vilar, C. (2016) Influence of Pyramids and Inverted Pyramids on Silicon Optical Properties. International Energy and Sustainability Conference (IESC), Cologne, 30 June-1 July 2016. https://doi.org/10.1109/IESC.2016.7569486

[28] Koshimizu, S. (2005) Key Engineering Materials, 291-292, 377-380. https://doi.org/10.4028/www.scientific.net/KEM.291-292.377

[29] Demichel, O. (2010) Propriétés Électroniques de Nanofils de Silicium obtenus par Croissance Catalysée. 14 janvier.

[30] Kobor, D., Tine, M., Hajjaji, A., Lebrun, L. and Guyomar, D. (2012) Journal of Modern Physics, 3, 402-409. https://doi.org/10.4236/jmp.2012.35056

[31] Abeles. F. (1950) Journal de Physique Radium, 11, 310-314. https://doi.org/10.1051/jphysrad:01950001107031000

[32] Tauc, J. (1966) The Optical Properties of Solids. Academic Press, New York.

[33] Kong, C.S. (2012) Formation of Silicon Nanowires by Chemical Vapour Deposition Technique Using Indium Catalyst. Master's Thesis, University of Malaya, Kuala Lumpur.

[34] Kovalev, D., Heckler, H., Ben-Chorin, M., Polisski, G., Schwartzkopff, M. and Koch, F. (1998) Physical Review Letters, 81, 2803. https://doi.org/10.1103/PhysRevLett.81.2803

[35] Chong-Jun, H. et al. (2012) Chinese Physics B, 21, 3. 